\title{
Smoothed Least-Laxity-First Algorithm for Electric Vehicle Charging: Online Decision and Performance Analysis with Resource Augmentation
}

\author{
Niangjun Chen, Member, IEEE, Christian Kurniawan, Yorie Nakahira, Member, IEEE, \\ Lijun Chen, Member, IEEE, Steven H. Low, Fellow, IEEE
}

\begin{abstract}
Adaptive charging can charge electric vehicles (EVs) at scale cost effectively, despite of the uncertainty in EV arrivals. We formulate adaptive EV charging as a feasibility problem that meets all EVs' energy demands before their deadlines while satisfying constraints in charging rate and total charging power. We propose an online algorithm, smoothed least-laxityfirst (sLLF), that decides the current charging rates without the knowledge of future arrivals and demands. We characterize the performance of the sLLF algorithm analytically and numerically. Numerical experiments with real-world data show that it has a significantly higher rate of feasible EV charging than several other existing EV charging algorithms. Resource augmentation framework is employed to assess the feasibility condition of the algorithm. The assessment shows that the sLLF algorithm achieves perfect feasibility with only a $7 \%$ increase in the maximal power supply of the charging station.
\end{abstract}

Index Terms-Power generation scheduling, Scheduling, Road vehicle power systems, Resource management, Battery chargers

\section{INTRODUCTION}

$\mathbf{T}$ HE proliferation of electric vehicles (EVs) is expected to accelerate for many years to come. EV charging at scale, however, presents a tremendous challenge as uncontrolled EV charging may strain the power grid and cause voltage instability. One way to mitigate the impact and stabilize the power grid as well as to manage uncertainty in the energy supply from renewable energy resources such as wind power and solar energy is by exploiting the flexibility in charging time and rate. To exploit and optimize this flexibility, many EV charging algorithms have been proposed and they can be categorized as either offline or online algorithms.

The offline algorithms require complete information on all EVs to decide the charging rates, e.g., [1]-[5]. Yet, information on future EV arrivals may not be available or expensive to obtain, which motivates the development of online algorithms,

N. Chen is with the Department of Information Systems Technology and Design, Singapore University of Technology and Design, Singapore 487372 He has join appointment with the Institute for High Performance Computing, Agency for Science, Technology, and Research, Singapore 138632. (e-mail: niangjun_chen@sutd.edu.sg, chennj@ihpc.a-star.edu.sg)

C. Kurniawan and Y. Nakahira are with the Department of Electrical and Computer Engineering, Carnegie Mellon University, PA 15213, USA. (e-mail: yorie@cmu.edu, christian.paryoto@gmail.com)

L. Chen is with the Department of Computer Science, University of Colorado Boulder, CO, 80309 USA. (e-mail: lijun.chen@ colorado.edu)

$\mathrm{S}$. H. Low is with the Department of Computing and Mathematical Sciences, California Institute of Technology, CA, 91125 USA. (e-mail: slow@caltech.edu) e.g., [6]-[11]. The optimum charging rate is obtained by solving an optimization problem in which various novel optimization techniques can be implemented (e.g., [12]-[15]), including linear programming approaches (e.g., [11], [16]). To reduce the computational complexity and memory usage, sorting or bisection based methods (e.g., earliest-deadline-first [17], [18], least-laxity-first [17], and Whittle's index policy [8], [19]) are often employed. These algorithms assume that the future information of the vehicle arrival is predictable and all of the instances are feasible. However, an online algorithm, which uses only information from EVs present at the charging station to decide their charging rates, may not produce a solution that satisfies all the constraints even when all EVs' demands can be satisfied. Thus, the efficacy of these online algorithms still depends on the accurate prediction of EV arrivals and energy demands that is difficult to obtain. Moreover, these algorithms require temporal coordination across time among a large number of EVs which is hard.

In view of these limitations, we investigated low-complexity $\mathrm{EV}$ charging that does not require prediction of $\mathrm{EV}$ arrivals/demands or temporal coordination. We first formulated the charging rate allocation as a feasibility problem to satisfy the energy demands of all EVs before their departure under constraints of individual maximum charging rate of every $\mathrm{EV}$ and the total available power supply. We then proposed an online algorithm, the smoothed least-laxity-first (sLLF), based on the classic least-laxity-first (LLF) with an improved success rate in achieving feasibility, that decides on the current charging rates based only on the information up to the current time. The sLLF algorithm makes the best possible decision by maximizing the minimum resulting laxity for the next time among the EVs currently in the system, so it can best accommodate arbitrary future EV arrivals. Here, laxity can be seen as the feasibility margin for EV charging and is defined as the EV's remaining time at the charging station decreased by the time needed to be fully charged at the maximum charging rate.

Cost related to the installation, replacement, and development of both the infrastructure of a charging station including power generation and the battery of an EV is also a factor to be considered in a charging algorithm [20]. Generally, the algorithm needs to adhere the limitation of the resources while still producing a feasible solution [13]. Thus, the feasibility condition of an algorithm can be assessed by characterizing the minimum amount of additional resources (i.e., total power 
supply and charging rates) that will allow the algorithm to produce a feasible solution, assuming all EVs' demands can be satisfied. In this study, the feasibility condition of the sLLF algorithm is analyzed using the resource augmentation framework. Resource augmentation is a prominent analysis framework for analyzing the performance of online algorithms for multiprocessor scheduling [21]-[27]. We apply this framework to the EV charging problem that can be viewed as a deadline scheduling problem by considering chargers as processors and EVs with certain energy demand as jobs. In our setting the power limit is time-varying, the maximum rates are heterogeneous, and the power limit may not necessarily be an integer multiplication of the maximum rate. To the best of our knowledge, our work is the first to analyze EV charging algorithms utilizing the resource augmentation framework. We also believe that this is the first extension of the framework into the cases for heterogeneous processors with a time-varying number of arrivals.

We further carried out numerical experiments using realworld datasets collected from Googles facilities in Mountain View (Google_mtv) and Sunnyvale (Google_svl) as well as the adaptive charging network (ACN) testbed we deployed at California Institute of Technology (Caltech), called CAGarage. At Caltech ACN, each EV arrives at a charger with energy demand and departure time. The charging facility also has a time-varying total power supply. The ACN performs real-time sensing, communication, and control using the profiles of each EV (including energy demand, departure time, and maximum charging rate) to decide the charging rate of each EV. See [28], [29] for more details on the Caltech infrastructure and [30] on the charging data.

The rest of the paper is organized as follows: Section II introduces the system model and proposes the sLLF algorithm; the performance of the SLLF algorithm is analyzed via the procedure describes in Section III; then the result and discussion of the performance analysis are presented in Section IV.

\section{Model AND Algorithm}

\section{A. System Model}

In this study, we consider a system with one charging station that serves a set of EVs, indexed by $i \in \mathcal{V}=\{1,2,3, \cdots\}$. We use a discrete-time model where time is divided into slots of equal sampling intervals, indexed by $t \in \mathcal{T}=\{0,1,2, \cdots, T\}$. $\mathrm{EV} i$ arrives at the charging station with an energy demand $e_{i}$ at time $a_{i}$, and departs from the station at time $d_{i}$. Each EV leaves at its departure time regardless of its charging conditions. We assume that there is an unlimited number of charging slots. However, we limit the availability of the power supply. This assumption is applicable for most slow chargers including ACN [28]. During its stay at the station, the EV is charged at a rate (or power) of $r_{i}(t) \geq 0, a_{i} \leq t<d_{i}$. For convenience, since the rate is adjusted at a discrete-time which make the value of the charging rate of vehicle $i$ at a given time is equal to the energy transmitted to the vehicle, we extend the definition of $r_{i}(t)$ to the entire temporal domain such that the notation $r_{i}(t)$ can be interpreted as both the charging rate of and the energy transmitted into the vehicle $i$ at time $t$. We denote the set of the remaining EVs in the charging station at time $t$ as $V_{t}=\left\{i \in A_{t}: a_{i} \leq t<d_{i}\right\}$ and the remaining energy demand of EV $i$ at time $t$ as $e_{i}(t)$. The notations are summarized in Table I.

To account for the limitations in the charger or battery of an EV, each EV $i$ can only be charged up to a peak rate, $\bar{r}_{i}$ :

$$
\begin{aligned}
& \begin{cases}0 \leq r_{i}(t) \leq \bar{r}_{i}, & t \in\left[a_{i}, d_{i}\right), i \in \mathcal{V} \\
r_{i}(t)=0, & t \notin\left[a_{i}, d_{i}\right), i \in \mathcal{V}\end{cases} \\
& \text { where } \quad \bar{r}_{\min } \leq \bar{r}_{i} \leq \bar{r}_{\max }, i \in \mathcal{V}
\end{aligned}
$$

To account for the limitations in the power grid or station, the charging station has a (possibly time-varying) power limit, $P(t)$, such that ${ }^{1}$

$$
\begin{array}{ll} 
& \sum_{i \in \mathcal{V}} r_{i}(t) \leq P(t), t \in \mathcal{T} \\
\text { where } & 0 \leq P_{\min } \leq P(t) \leq P_{\max }
\end{array}
$$

Finally, every EV's energy demands need to be satisfied ${ }^{2}$

$$
\sum_{t \in \mathcal{T}} r_{i}(t)=e_{i}, i \in \mathcal{V}
$$

The charging problem instance is then defined as a quintuple

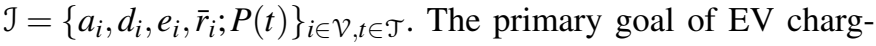
ing is to satisfy every EV's energy demands under the above power supply and peak charging rate constraints (Eqs. (1)-(3)).

Definition 1 (feasible instance). An EV charging problem instance $\mathcal{J}$ is offline feasible if there exist charging rates $r=\left\{r_{i}(t): i \in \mathcal{V}, t \in \mathcal{T}\right\}$ that satisfy Eqs. (1)-(3).

Because Eqs. (1)-(3) are affine, verifying the feasibility of an EV charging instance is a linear programming (LP) problem for which many efficient algorithms exist.

\begin{tabular}{cl} 
& \multicolumn{1}{c}{ TABLE I } \\
& \\
NOTATIONS \\
\hline notation & \multicolumn{1}{c}{ description } \\
\hline \hline $\mathcal{V}$ & set of EVs in the system \\
$V_{t}$ & set of EVs remain in the charging station at time $t$ \\
$\mathcal{T}$ & set of the system's discrete-time \\
$a_{i}$ & arrival time of EV $i$ \\
$d_{i}$ & departure time of EV $i$ \\
$e_{i}$ & energy demand of EV $i \in \mathcal{V}$ \\
$e_{i}(t)$ & remaining energy demand of EV $i$ at time $t \in \mathcal{T}$ \\
$r_{i}(t)$ & charging rate of or energy transmitted into EV $i$ at time $t$ \\
$P(t)$ & power limit of the charging station at time $t$ \\
$\mathcal{J}$ & EV charging problem instance \\
\hline
\end{tabular}

\section{B. Online Scheduling}

In practice, information of the energy demand and departure time of an EV is only available after its arrival. Consequently, the charging station need to employ an online algorithm to determine the current charging rate of EV $i$ at time $t, r_{i}(t)$,

${ }^{1}$ All EVs at the charging station can be simultaneously charged as long as Eqs. (1) and (2) are satisfied.

${ }^{2}$ The actual constraint in $\mathrm{ACN}$ is $\sum_{t \in \mathcal{T}} \delta r_{i}(t)=e_{i}, i \in \mathcal{V}$, where $\delta$ is the sojourn time in unit of hours of sampling time intervals, $e_{i}$ has unit of $k W h$, $r_{i}(t)$ has unit of $k W$ [28]. Since $r_{i}(t)$ can always be rescaled according to $\delta$, we set $\delta=1$ without loss of generality. 
given information only up to the current time by mapping it into

$$
\begin{aligned}
\mathcal{J}_{t} & =\left\{a_{i}, d_{i}, e_{i}(\tau), \bar{r}_{i} ; P(\tau)\right\}_{i \in \mathcal{V}_{t}, \tau \leq t} \\
\text { where } \quad e_{i}(\tau) & =e_{i}-\sum_{j=0}^{\tau-1} r_{i}(j)
\end{aligned}
$$

Definition 2 (online algorithm). An online algorithm is a sequence of functions, $\mathcal{A}=\left\{\mathcal{A}_{t}\right\}$, where each function, $\mathcal{A}_{t}$ : $\mathrm{J}_{t} \rightarrow r(t)$, maps the information up to the current time, $\mathcal{J}_{t}$, to the current charging rates, $r(t)=\left\{r_{i}(t)\right\}_{i \in \mathcal{V}_{t}}$.

Definition 3 (feasibility of an algorithm). An (online) algorithm, $\mathcal{A}$, is (online) feasible on instance $\mathcal{J}$ if it gives charging rates that satisfy constraints defined in Eqs. (1)-(3).

For an online algorithm to be feasible, it must be online feasible for all offline feasible instances. However, In general, there does not exist an online algorithm that is feasible on all offline feasible instances. In this paper, we investigate the cases in which online feasibility can be guaranteed with additional assumptions.

\section{Smoothed Least-Laxity-First Algorithm}

Our proposed online algorithm, the sLLF algorithm, is an improvement from the classic LLF algorithm [31]. We can see laxity as a measure for the flexibility (or urgency) in the charging process of an EV.

Definition 4 (laxity). The laxity of an $E V i \in \mathcal{V}$ at time $t \in \mathcal{T}$ is defined as the remaining time of the vehicle in the charging station minus the minimum remaining time needed to be fully charged $^{3}$ i.e.,:

$$
\ell_{i}(t)= \begin{cases}{\left[d_{i}-t\right]^{+}-\frac{e_{i}(t)}{\bar{r}_{i}},} & t \geq a_{i} \\ +\infty, & t<a_{i}\end{cases}
$$

where $[\cdot]^{+}$denotes as the projection onto the set $\mathscr{R}_{+}$of nonnegative real numbers.

Notice that for $t<d_{i}, \ell_{i}(t+1)=\ell_{i}(t)-\left(1-\frac{r_{i}(t)}{\bar{r}_{i}}\right)$. Here, we can rewrite Eq. (1) as $0 \leq \frac{r_{i}(t)}{\bar{r}_{i}} \leq 1$ which suggests that laxity of $\mathrm{EV} i$ is monotonically decreasing at $a_{i} \leq t<d_{i}$. Then, Eq. (3) implies that, for $t \geq d_{i}, e_{i}(t)=0, i \in \mathcal{V}$ which means that for all $\left.t \geq d_{i}, \ell_{i}(t)=\left[d_{i}-t\right)\right]^{+}-\frac{e_{i}(t)}{\bar{r}_{i}}=0$. Therefore, the feasibility conditions from Definition 3 implies that:

Proposition 1. The algorithm $\mathcal{A}$ that satisfy constraints (1) and (2) is feasible on an instance $\mathcal{J}$ if and only if $\mathcal{A}$ gives charging rates that result in non-negative laxity for all EVs, i.e., $\ell_{i}(t) \geq 0, \forall i \in \mathcal{V}, t \in \mathcal{T}$.

Proposition 1 suggests that the smallest laxity among all EVs can serve as a measure of the distance from infeasibility. A naive approach is to charge EVs starting from those with the least laxity, i.e., the LLF algorithm. However, the LLF algorithm may compromise the feasibility of certain offline feasible instances (see Section IV). The LLF algorithm also

\footnotetext{
${ }^{3}$ For convenience, laxity is defined on the whole temporal domain $\mathcal{T}$.
}

cause excessive preemption and oscillations in the charging rate $^{4}$, which may reduce the lifetime of certain batteries (e.g., Li-ion) [10]. To eliminate these drawbacks, we propose an alternative approach by maximizing the minimum laxity among all EVs with the charging rate at time $T, r(T)=$ $\left\{r_{i}(T): \forall i \in \mathcal{V}\right\}$, as the design variable in order to maximize the feasibility margin, $\max _{r(T)} \min _{i \in \mathcal{V}} \ell_{i}(T)$.

However, because the solution to the above maximization problem may not be unique, we considered the following problem to produce a unique solution:

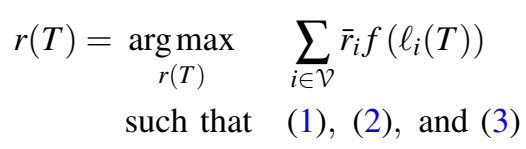

where $f$ is any function that is twice continuously differentiable, strictly concave, and monotonically increasing. Here, if an instance $\mathcal{J}$ is offline feasible, then there exists certain charging rates that achieve $\ell_{i}(T)=0, \forall i \in \mathcal{V}$, which yields $\sum_{i \in \mathcal{V}} f\left(\ell_{i}(T)\right)=\sum_{i \in \mathcal{V}} f(0)$. Since the laxity is monotonically decreasing at any $t \in \mathcal{T}$, such charging rates also satisfy Proposition 1 which implies that Problem (5) is feasible on instance J, i.e.,:

Remark 1. Problem (5) is feasible for any offline feasible instance.

Notice that Problem (5) is an offline problem that requires the information of all vehicles. To obtain an (online) solution without the information of incoming EVs, we approximate (5) using the available causal information, by successively maximizing the laxity

$$
\begin{array}{ll}
\max _{r(t)} & \sum_{i \in \mathcal{V}} \bar{r}_{i} f\left(\ell_{i}(t+1)\right) \\
\text { such that } & (1),(2), \text { and } r_{i}(t) \leq e_{i}(t), i \in \mathcal{V}_{t}
\end{array}
$$

at each time $t \in \mathcal{T}$ for given $\ell_{i}(t), i \in \mathcal{V}_{t}{ }^{5}$ Problem (6) also maximizes the minimum laxity at time $t+1, \min _{i \in \mathcal{V}_{t}} \ell_{i}(t+1)$, and thus maximizes the feasibility margin at time $t$.

To solve Problem (6), we first need to look at the KarushKuhn-Tucker (KKT) conditions of the problem:

$$
\begin{array}{ll}
r_{i}(t) \geq 0 & i \in \mathcal{V}_{t} \\
r_{i}(t) \leq \min \left(e_{i}(t), \bar{r}_{i}\right) & i \in \mathcal{V}_{t} \\
\sum_{i \in \mathcal{V}_{t}} r_{i}(t) \leq P(t) & i \in \mathcal{V}_{t} \\
f^{\prime}\left(\ell_{i}(t+1)\right)+\bar{\lambda}_{i}-\underline{\lambda}_{i}+\mathcal{V}=0 & i \in \mathcal{V}_{t} \\
\underline{\lambda}_{i} \geq 0, \bar{\lambda}_{i} \geq 0, \mathcal{V} \geq 0 & i \in \mathcal{V}_{t} \\
\underline{\lambda}_{i} r_{i}(t)=0, \bar{\lambda}_{i}\left\{r_{i}(t)-\min \left(e_{i}(t), \bar{r}_{i}\right)\right\}=0 & i \in \mathcal{V}_{t}
\end{array}
$$

${ }^{4}$ For example, consider a system of two EVs, where $\ell_{1}(0)=1.25, \ell_{2}(0)=$ 0.75 and $\bar{r}_{1}=\bar{r}_{2}=P(t)=1, t \in \mathcal{T}$. EV 1 and EV 2 will be charged according to $\left(r_{1}(0), r_{2}(0)\right)=(0,1),\left(\ell_{1}(1), \ell_{2}(1)\right)=(0.25,0.75) ;\left(r_{1}(1), r_{2}(1)\right)=(1,0)$, and so on. In this example, both EV switches in-between charging and not charging.

${ }^{5}$ For more complex form of power limits, in Problems (5) and (6), the power constraints in Eq. (2) can be replaced by $\operatorname{Ar}(t) \leq_{\text {e.w. }} P(t)$, for element-wise inequality and positive matrix $A$. Remark 1 also holds for $\operatorname{Ar}(t) \leq_{\text {e.w. }} P(t)$. 
where $\underline{\lambda}_{i}, \bar{\lambda}_{i}$, and $v$ are the dual variables for constraints (7), (8), and (9) respectively. Consider three mutually exclusive cases:

- $r_{i}(t)=0$ that leads to $\bar{\lambda}_{i}=0$ and

$$
\frac{r_{i}(t)}{\bar{r}_{i}}=f^{\prime-1}(-v)-\ell_{i}(t)+1-\underline{\lambda}_{i} \leq f^{\prime-1}(-v)-\ell_{i}(t)+1
$$

- $r_{i}(t) \in\left\{0, \min \left(e_{i}(t), \bar{r}_{i}\right)\right\}$ which implies $\bar{\lambda}_{i}=\underline{\lambda}_{i}=0$; obtained from (12) (complementary slackness). Substituting $\bar{\lambda}_{i}=\underline{\lambda}_{i}=0$ into (10), we obtain

$$
\frac{r_{i}(t)}{\bar{r}_{i}}=f^{\prime-1}(-v)-\ell_{i}(t)+1
$$

- $r_{i}(t)=\min \left(e_{i}(t), \bar{r}_{i}\right)$ that leads to $\underline{\lambda}_{i}=0$ and

$$
\frac{r_{i}(t)}{\bar{r}_{i}}=f^{\prime-1}(-v)-\ell_{i}(t)+1+\hat{\lambda}_{i} \geq f^{\prime-1}(-v)-\ell_{i}(t)+1
$$

Here, the inverse of $f^{\prime}$ exists because $f^{\prime}$ is strictly concave, strictly increasing, and twice continuously differentiable.

Defining an variable $L(t)=f^{\prime-1}(-v)$, the following can be obtained:

Proposition 2. With $f$ strictly concave, strictly increasing, and twice continuously differentiable, a solution to (6) can be obtained by combining Eqs. (13)-(15):

$$
r_{i}^{*}(t)=\left[\bar{r}_{i}\left(L(t)-\ell_{i}(t)+1\right)\right]_{0}^{\min \left(\bar{r}_{i}, e_{i}(t)\right)}, \quad i \in \mathcal{V}_{t}
$$

where $[x]_{a}^{b}$ denotes the projection of the scalar $x$ on interval $[a, b]$ and $r_{i}^{*}(t)$ is the resulted $r_{i}(t)$. The solution is then attained at the boundary

$$
\begin{aligned}
\sum_{i \in \mathcal{V}_{t}} r_{i}^{*}(t) & =\sum_{i \in \mathcal{V}_{t}}\left[\bar{r}_{i}\left(L(t)-\ell_{i}(t)+1\right)\right]_{0}^{\min \left(\bar{r}_{i}, e_{i}(t)\right)} \\
& =\min \left(P(t), \sum_{i \in \mathcal{V}_{t}} \min \left(\bar{r}_{i}, e_{i}(t)\right)\right)
\end{aligned}
$$

For EV $i \in \mathcal{V}_{t}$ with $\bar{r}_{i} \leq e_{i}(t)$, we obtain charging rate from Eq. (16) that makes $\ell_{i}(t+1)=[L(t)]_{\ell_{i}(t)-1}^{\ell_{i}(t)}$. Hence, $L(t)$ can be considered as a threshold of $\ell_{i}(t+1)$. Since $r_{i}^{*}(t)$ in Eq. (16) is an increasing function of $L(t)$, a binary search can be employed to find the threshold $L(t)$ in Eq. (17). Given $L(t)$, the charging rates $r_{i}^{*}(t), i \in \mathcal{V}_{t}$ is then determined using Eq. (16). This procedure is a scalable algorithm that we formally state in Algorithm 1, and name it as the smoothed least-laxity-first $(s L L F)$ algorithm.

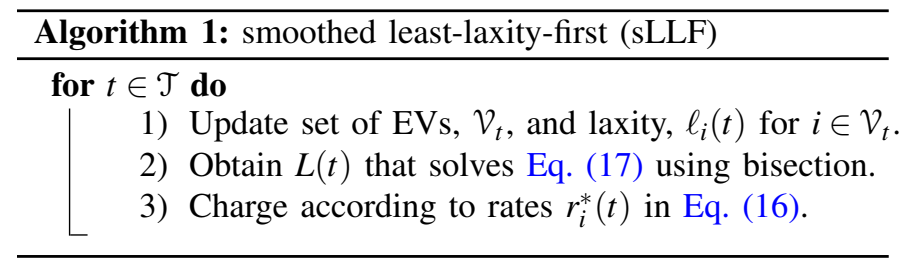

We found that the computational complexity of this SLLF algorithm at each time $t$ is $O\left(\left|\mathcal{V}_{t}\right|+\log \left(\frac{1}{\delta}\right)\right)$, where $\delta$ is the level of tolerable error. We need $O\left(\left|\mathcal{V}_{t}\right|\right)$ operations to update the laxity of vehicles, and $O\left(\log \left(\frac{1}{\delta}\right)\right)$ operations for binary search for $L(t)$. We also note that the sLLF algorithm possesses the following properties:

1) Persistence

Lemma 1. Under the sLLF algorithm, if there exist two $E V s i, j \in \mathcal{V}$ such that

$$
\ell_{i}(t) \leq \ell_{j}(t) \text { but } \ell_{i}(t+1)>\ell_{j}(t+1)
$$

then either one of the following holds:

$$
\left\{\begin{array}{l}
t \geq d_{i} \& r_{i}(t)=0 \\
t<d_{i} \& t<d_{j} \& e_{j}(t+1)=0 \& r_{i}(t) \neq 0
\end{array}\right.
$$

Proof of this Lemma can be found in [32, Section II-C].

2) Fairness

From Lemma 1, the solution of (6) does not depend of the specific choice of the value function $f$ as long as $f$ is concave, strictly increasing, and has a derivative whose inverse function is well-defined. Without loss of generality, we consider $f(x)=\log (x)$. Since non-negative weighted sum and composition with an affine mapping preserve concavity, $C(r(t))=$ $\sum_{i \in \mathcal{V}_{t}} \bar{r}_{i} f\left(l_{i}(t)-1+\frac{r_{i}(t)}{\bar{r}_{i}}\right)$ is a concave function of $r(t)=\left[r_{1}(t), r_{2}(t), \cdots, r_{\left|\mathcal{V}_{t}\right|}(t)\right]^{T}$. Let $\hat{r}(t) \neq r(t)$ be any rates that satisfy constraints in Eqs. (1) and (2), where $\hat{\ell}_{i}(t), \quad i \in \mathcal{V}_{i}$ be the resultant laxity, then, from the first-order-condition of concave functions, $C(\hat{r}(t))-$ $C(r(t))+(r(t)-\hat{r}(t))^{T} \nabla C(r(t)) \geq 0$. Since $r(t)$ is the optimal solution, then

$$
\begin{aligned}
0 & \leq C(r(t))-C(\hat{r}(t)) \leq(r(t)-\hat{r}(t))^{T} \nabla C(r(t)) \\
& \leq \sum_{i \in \mathcal{V}_{t}} \bar{r}_{i} \frac{\ell_{i}(t)-\hat{\ell}_{i}(t)}{\ell_{i}(t+1)}
\end{aligned}
$$

where the derivative above is taken over $r(t)$ for $f\left(\ell_{i}(t+1)\right)=\log \left(\ell_{i}(t+1)\right)$. On the other hand, if $\hat{\ell}_{i}(t+$ $1)>\ell_{i}(t+1)$ for some EV $i \in \mathcal{V}_{t}$, then $r_{i}(t)<\hat{r}_{i}(t) \leq \bar{r}_{i}$. This can only happen when $\ell_{i}(t+1) \geq L(t)$ or $\ell_{i}(t+1)>$ $L(t)$. As EV $i$ in $\hat{r}_{i}(t)$ receives more energy than that in $r_{i}(t)$, there exists an EV $j$ that receives less energy in $\hat{r}_{j}(t)$. Any EV $j$ that receives non-zero energy satisfies $\hat{\ell}_{j}(t+1) \leq L(t+1)=\ell_{i}(t+1)$ and $\hat{\ell}_{j}(t+1)<\ell_{j}(t+1)$. Corollary 1. Given the past charging rate $r^{t-1}$, the $s L L F$ algorithm finds a current charging rate $r(t)$ that is both proportionally and max-min fair to one-step-ahead laxity $\ell_{i}(t+1)$ with $\hat{\ell}_{i}(t+1)$ as another laxity produced by a charging rate satisfying the constraints in (6):

- weighted proportional fairness:

$$
\sum_{i \in \mathcal{V}_{t}} \bar{r}_{i} \frac{\hat{\ell}_{i}(t+1)-\ell_{i}(t+1)}{\ell_{i}(t+1)} \leq 0
$$

- max-min fairness: if $\hat{\ell}_{i}(t+1)>\ell_{i}(t+1)$ for some $E V i \in V_{t}$, then there exits $E V j \in V_{t}$ such that 


$$
\hat{\ell}_{j}(t+1) \leq \ell_{i}(t+1) \text { and } \hat{\ell}_{j}(t+1)<\ell_{j}(t+1)
$$

\section{Performance Analysis}

We compare the performance of our proposed SLLF algorithm with several common scheduling algorithms and assess its feasibility condition utilizing the resource augmentation framework.

\section{A. Resource Augmentation Framework}

There are two extreme cases in which online algorithms can be feasible for any offline feasible instances: $\bar{r}_{i} \rightarrow \infty$ $\forall i \in \mathcal{V}$ and $P(t) \rightarrow \infty$. In the first case, $\bar{r}_{i} \rightarrow \infty \forall i \in \mathcal{V} \equiv$ $P(t) \leq \min _{i \in \mathcal{V}_{t}} \bar{r}_{i} \forall t \in \mathcal{T}$, the charging problem is identical to the single processor preemptive scheduling problem where the processing capacity is time-variant. Here, the earliestdeadline-first (EDF) algorithm is feasible for any offline feasible instances [18]. In the second case, $P(t) \rightarrow \infty \equiv P(t) \geq$ $\sum_{i \in V_{t}} \bar{r}_{i}(t) \forall t \in \mathcal{T}$, the sLLF algorithm is feasible for any offline feasible instances. Beyond these two extreme cases, no online algorithm can be feasible on all offline feasible instances [17].

From the two cases mentioned above, we can observe that if more resources (e.g., $P(t)$ and $\left.\bar{r}_{i}\right)$ are allowed, an otherwise infeasible problem may become online feasible under an online algorithm. Based on this, we performed a resource augmentation study to characterize the minimum amount of additional resources that will allow an algorithm to produce a feasible solution. Specifically, we analyzed the performance of the sLLF algorithm by adding more (minimum) either power supply (power augmentation) or both power supply and peak charging rate (power+rate augmentation). The former augmentation allows more EVs to be charged simultaneously, while the latter allows faster charging. These two augmentation approaches are qualitatively different and provide complementary insights into the behavior of the sLLF algorithm. In our setting, the power limit is time-varying, the maximum rates are heterogeneous, and the power limit may not necessarily be integer multiplication of the maximum rate. Consequently, value of augmentation also depends on the interplay between $P_{\min }, P_{\max }, \bar{r}_{\min }$, and $\bar{r}_{\max }$, which complicates the analysis.

1) Power Augmentation: In this augmentation, we allowed online algorithm to utilize $\varepsilon$ more power such that

$$
P^{\text {online }}(t)=(1+\varepsilon) P(t) \text { but } \bar{r}_{i}^{\text {online }}=\bar{r}_{i}
$$

We will call this augmentation as $\varepsilon$-power augmentation, where

Definition 5. [ $\varepsilon$-power augmented instance] Given an EV

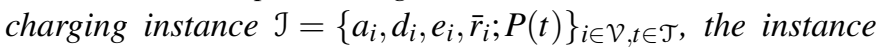
under $\varepsilon$-power augmentation is defined as $\left\{a_{i}, d_{i}, e_{i}, \bar{r}_{i} ;(1+\right.$ ع) $P(t)\}_{i \in \mathcal{V}, t \in \mathcal{T}}$.

Definition 6 ( $\varepsilon$-power feasibility). An online algorithm $\mathcal{A}$ is $\varepsilon$-power feasible if $\mathcal{A}$ is feasible on the $\varepsilon$-power augmented instances $\mathcal{J}_{p}(\varepsilon)$ generated from any offline feasible instance J. ${ }^{6}$

Unfortunately, there is no online algorithm that $\varepsilon$-power feasible for any finite $\varepsilon>0[22]^{7}$. However, under a mild assumption, the $\varepsilon$-power feasibility condition can be obtained for a finite $\varepsilon$. Assume that the energy demand of each EV is bounded by $X$ and that the inter-arrival time between consecutive arrivals is greater than $N$, i.e.,:

$$
e_{i} \leq X \text { and }\left|a_{i}-a_{j}\right|>N ; i, j \in \mathcal{V}
$$

where, the value of $N$ can be controlled by choosing appropriate sojourn time for a sampling intervals (the shorter the sojourn time, the smaller the value of $N$ ) and the value of $X$ can be obtain from maximum battery capacity for common EVs. Then, it can be proven (see [32, Appendix A]) that we can characterize the relation between $N$ and the sufficient amount of resource augmentation $\varepsilon$ as follows:

Theorem 1. If both conditions in (22) hold, then the sLLF algorithm is $\varepsilon$-power feasible with

$$
\varepsilon=\frac{P_{\max }}{P_{\min }}\left(\log _{\varphi}\left(\frac{\sqrt{5} X}{N P_{\max }}+\frac{1}{2}\right)+2\right)-1
$$

where $\varphi \approx 1.61803$ is the golden ratio.

Now, if the inter-arrival time is equal to $N$ and the power demand is equal to $X$, then the incoming energy demand per unit time is $\frac{X}{N}$. Since the total power supply is $P_{\max }$ per unit time, $N$ should be at least $\frac{X}{P_{\max }}$ for offline feasibility which is a mild assumption. With this, we then can apply a special condition to Theorem 1:

Corollary 2. For constant power limit $P(t)=P, t \in \mathcal{T}$, and $N \geq \frac{X}{P_{\max }}$, then

$$
\varepsilon \leq \log _{\varphi}\left(\sqrt{5}+\frac{1}{2}\right)+1 \approx 3.091639884
$$

Corollary 2 is obtained from substituting $P_{\max }=P_{\min }=P$ into the bound of Theorem 1, and upper bounding using $N \geq$ $\frac{X}{P}$. From Corollary 2 , the sLLF algorithm is approximately 3 power feasible. Thus, under this condition, theoretically, the sLLF algorithm can achieve $100 \%$ feasibility by increasing the available power supply three times.

2) Power+Rate Augmentation: In this case, online algorithm is allowed to utilize $\varepsilon$ more power and higher maximum charging rate such that

$$
P^{\text {online }}(t)=(1+\varepsilon) P(t) \text { and } \bar{r}_{i}^{\text {online }}=(1+\varepsilon) \bar{r}_{i}
$$

\footnotetext{
${ }^{6}$ Alternatively, the (minimum) value of $\varepsilon$ can also be interpreted as the constraints on instances that are online feasible. That is, given the original resource $P(t), \bar{r}_{i}(t)$, the algorithm is online feasible for any instances $\mathcal{J}=\left\{a_{i}, d_{i}, e_{i}, \bar{r}_{i} ; P(t) /(1+\varepsilon)\right\}_{i \in \mathcal{V}, t \in \mathcal{T}}$ that is offline feasible given the reduced resource $P(t) /(1+\varepsilon), \bar{r}_{i}(t)$. Large $\varepsilon$ restricts possible instances, thus less likely to be online infeasible.

${ }^{7}$ It is shown in [22] that the LLF algorithm is not $\varepsilon$-power feasible for any $\varepsilon>0$ for uniform processors and time-invariant number of processors. Since their setting is a special case of our setting, the same results extend to our setting.
} 
We will call this augmentation as $\varepsilon$-power+rate augmentation, where

Definition 7. [E-power+rate augmented instance] Given an EV charging instance $\mathcal{J}=\left\{a_{i}, d_{i}, e_{i}, \bar{r}_{i} ; P(t)\right\}_{i \in \mathcal{V}, t \in \mathcal{T} \text {, we }}$ define the $\varepsilon$-power+rate augmented instance as $\left\{a_{i}, d_{i}, e_{i},(1+\right.$ $\left.\varepsilon) \bar{r}_{i} ;(1+\varepsilon) P(t)\right\}_{i \in \mathcal{V}, t \in \mathcal{T}}$.

Definition 8 ( $\varepsilon$-power+rate feasibility). An online algorithm $\mathcal{A}$ is $\varepsilon$-power+rate feasible if $\mathcal{A}$ is feasible on the $\varepsilon$-power+rate augmented instances $\mathcal{J}_{p r}(\varepsilon)$ generated from any offline feasible instance $\mathrm{J}$.

However, unlike the case of power augmentation, without any assumptions of the arrival patterns, the following apply [32, Appendix B]:

Theorem 2. The sLLF algorithm is $\varepsilon$-power+rate feasible with

$$
\varepsilon=\max _{i \in \mathcal{V}}\left(\max _{\tau_{1}, \tau_{2} \in\left[a_{i}, d_{i}\right]} \frac{P\left(\tau_{1}\right)}{P\left(\tau_{2}\right)}-\max _{\tau \in\left[a_{i}, d_{i}\right]} \frac{\bar{r}_{i}}{P(\tau)}\right)
$$

Here, solving the maximization problem in Theorem 2, theoretically, the sLLF algorithm will achieve a 100\% feasibility with an $\varepsilon$ increase in both available power supply and maximum charging rate.

\section{B. Experimental Setup}

We employed trace-based simulation on real EV datasets from the ACN deployment (CAGarage) as well as Google's facilities in Mountain View (Google_mtv) and Sunnyvale (Google_svl) to evaluate the performance of our proposed algorithm. The datasets contain a total of 52,362 charging sessions over more than 4,000 charging days in 2016 at 104 locations (Table II provides a summary of the data), an instance consists of one charging day. We compute the minimum power capacity in which each instance is feasible by using an offline LP, i.e., we minimize $P(t)$ subject to Eqs. (1)(3), which corresponds to the minimum power supply for the instance to be offline feasible. We used this minimum power supply to generate an offline instance and tested if the instance is feasible under an online algorithm.

TABLE II

\begin{tabular}{|l|c|c|c|}
\hline datasets & instances & EV sojourn time (min) & laxity (min) \\
\hline \hline CAGarage & 92 & $321(11,720)$ & $231(0.1,660)$ \\
\hline Google_mtv & 3793 & $149(10,720)$ & $35(0.001,694)$ \\
\hline Google_svl & 246 & $152(11,720)$ & $38(0.02,676)$ \\
\hline
\end{tabular}

Statistics of the EV charging instances that show the average of the sojourn times and laxity in minutes unit; the minimum and maximum values are indicated inside the brackets.

We compared the performance of the sLLF algorithm against several common heuristic online scheduling algorithms: earliest-deadline-first (EDF) [17], [18], least-laxityfirst (LLF) [17], equal/fair share (ES) [33], remaining energy proportional (REP) [18], and an online linear program (OLP) [11]. The implementation of these algorithms for the current problem is summarized in [32, Section III-B].

\section{Performance Evaluation and Comparison}

In this section, we will evaluate the performance of the SLLF algorithm without (Section IV-A) and with (Section IV-B) resource augmentation. For this purpose, we define the success rate of an algorithm as:

Definition 9 (success rate). The success rate of an algorithm is the ratio of online feasible instances under the algorithm to all existing instances.

\section{A. Without Resource Augmentation}

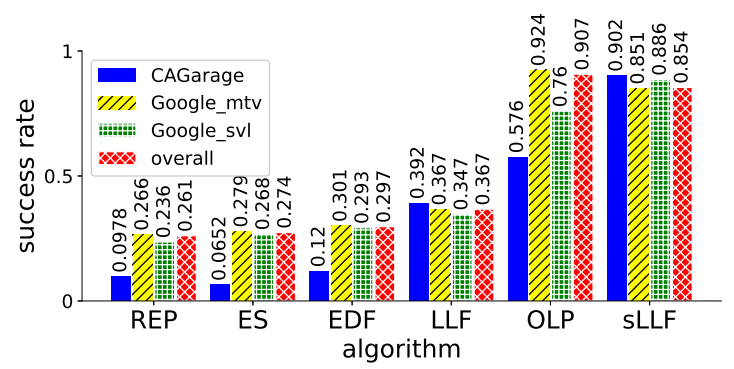

Fig. 1. Bar chart showing the success rate of the utilized algorithms in finding a feasible online schedule from different datasets without resource augmentation. The displayed values are rounded to three significant figures. (a)

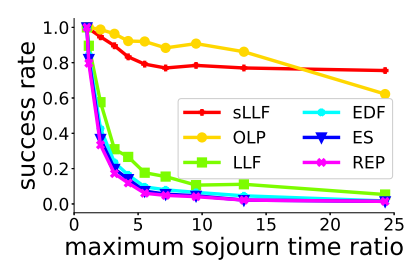

(c) power augmentation

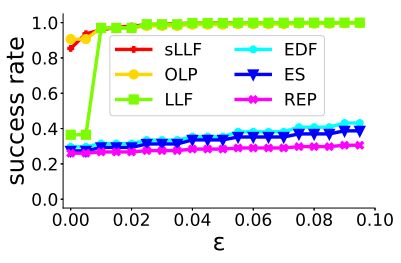

(b)

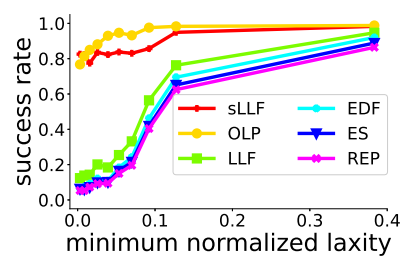

(d) power+rate augmentation

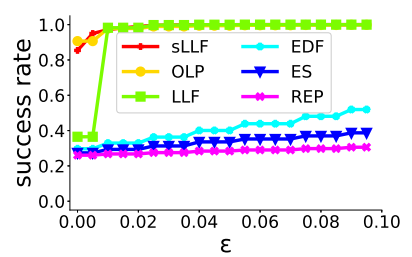

Fig. 2. Plots of success rate in finding a feasible online schedule without (top) and with (bottom) resource augmentation.

Comparing the success rate of the sLLF algorithm against different algorithms, we found that our proposed algorithm achieves a more uniform high success across different datasets (see Fig. 1). From Fig. 1 we can also see that the EDF, ES, and REP algorithms perform much worse in terms of finding feasible schedules as expected because these algorithms do not consider the deadline, maximum charging rate, and remaining energy of each EV simultaneously which are necessary to find the feasibility. We can also see that, despite its similarity, the LLF algorithm achieve lower success rate than the SLLF algorithm that suggests the importance of maximizing minimum 
laxity to eliminate the infeasibility of certain offline feasible instances in the LLF algorithm (see Section II-C).

Moreover, although the OLP algorithm achieves a higher success rate in finding a feasible online schedule from Google_mtv dataset, it requires solving LP problem at every time-step. With the currently available LP solver, the computational complexity for solving LP problem of size $\mathbf{n}$ will be greater than $O\left(\mathbf{n}^{2}\right)$ [34]. Thus, at every given time $t$ the OLP algorithm has computational complexity higher than $O\left(\left|\mathcal{V}_{t}\right|^{2}\right)$ which is computationally more expensive than the sLLF algorithm that has a complexity of $O\left(\left|\mathcal{V}_{t}\right|+\log \left(\frac{1}{\delta}\right)\right)$.

Furthermore, we observed that the minimum normalized laxity and the maximum ratio between EV sojourn times have high correlations with the success rate of the algorithms. Here, the maximum ratio between $\mathrm{EV}$ sojourn times is defined as the maximum ratio between the longest and shortest EV sojourn times in the instances while the minimum normalized laxity of an EV is defined as the laxity divided by the EV sojourn times, $\frac{\ell_{i}\left(a_{i}\right)}{\left(d_{i}-a_{i}\right)}$. To study this, we categorized the dataset we have into different sets and the success rate of the algorithms based on these different data categories can be found in Figs. 2a and $2 b$.

Fig. 2a shows that as the maximum ratio between EV sojourn times increases, all algorithms considered have decreased success rates. This indicates that a large degree of variation in the sojourn time may decrease the performance of online scheduling algorithms. From the Fig. $2 \mathrm{a}$ we can also see that the sLLF algorithm is least sensitive to the changing of the maximum ratio between EV sojourn times while still maintaining a high success rate. This indicates a potential benefit of the sLLF algorithm against a large variation of EVs' sojourn time that common in real-world applications. Meanwhile, Fig. $2 b$ shows that higher minimum normalized laxity improves the algorithms' success rate which implies that shorter sojourn time is more desirable to improve the performance of the scheduling algorithms. The result indicates that larger laxity gives a greater advantage in the scheduling system which is expected as a less urgent environment is easier to maintain. As we can see in Fig. 2b, the sLLF algorithm has one of the highest success rates for all minimum normalized laxity even when the minimum normalized laxity is small. This implies another potential benefit of the sLLF algorithm in a high urgency scheduling environment such as in some public charging stations.

\section{B. With Resource Augmentation}

We analyzed the performance of the online algorithms with resource augmentation in a) power supply and b) both power supply along with peak charging rate to gain further insight into the algorithms' behavior (see Figs. 2c and 2d). As expected, the success rate of all algorithms increase with more available resources that suggest the benefit of higher power supply and/or peak charging rate in the scheduling system. Although the performance of the sLLF algorithm in the event without resource augmentation is lower than the OLP algorithm, it can achieve a 0.95 success rate with only a 0.02 increase in resources.
TABLE III

\begin{tabular}{|l|c|c|c|c|c|c|}
\hline \multirow{2}{*}{ augmentation } & \multicolumn{6}{|c|}{$\varepsilon$} \\
\cline { 2 - 7 } & REP & ES & EDF & LLF & OLP & sLLF \\
\hline \hline power supply & 4.61 & 3.65 & 1.39 & 0.07 & 0.28 & 0.07 \\
\hline $\begin{array}{l}\text { power supply and } \\
\text { peak charging rate }\end{array}$ & 4.61 & 3.24 & 0.54 & 0.05 & 0.28 & 0.05 \\
\hline
\end{tabular}

Minimum resource augmentation to achieve a perfect success rate in finding a feasible online schedule for all instances.

Inspecting further, we listed in Table III the minimum resource augmentation required for each algorithm to achieve $100 \%$ feasibility for all instances. From the table, we can see that, together with the LLF algorithm, our sLLF algorithm has the smallest $\varepsilon$ among the algorithms considered. The proposed algorithm can achieve perfect feasibility using only 0.07 power augmentation which is significantly smaller than the predicted value in Corollary 2. Thus, our proposed algorithm has the potential in reducing the infrastructure cost for EV charging facility which will also be beneficial in an application where the resources are limited. (a) LLF

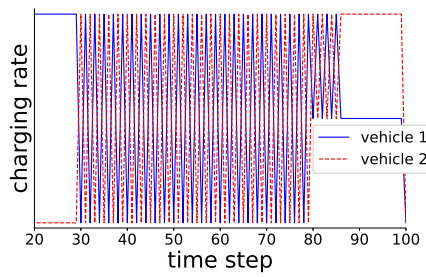

(b) SLLF

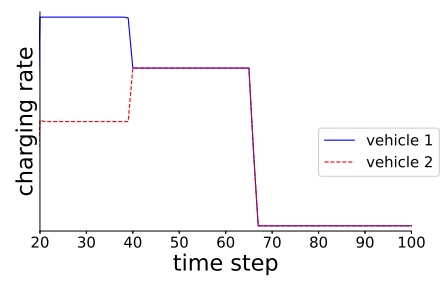

Fig. 3. Charging rate from two vehicles at each time step of a hypothetical case that exaggerate the oscillation behaviour in the LLF algorithm obtained using (a) the LLF and (b) the sLLF algorithms.

Additionally, although the LLF and sLLF algorithms have equal $\varepsilon$ feasibility, the sLLF algorithm, as implies by Lemma 1, does not exhibit undesirable oscillations behavior such as can be found in the LLF algorithm (Footnote 4). To inspect this property, we simulated a hypothetical case that exaggerate the oscillation behavior in the LLF algorithm. In the simulation we introduced two vehicles with equal maximum charging rates that arrive and will depart at the same time where there isn't other vehicle present at the charging station. The charging rate at each time step of this simulation obtained using the LLF and sLLF algorithms can be seen in Figs. 3a and $3 \mathrm{~b}$ respectively. The result, agrees with Lemma 1 , shows that the sLLF algorithm eliminates the oscillation behavior that can reduce the lifetime of certain batteries.

\section{CONClusion}

In this work, we formulated EV charging as a feasibility problem that meets all EVs' energy demands before departure under the individual charging rate of each $\mathrm{EV}$ and the total power resource constraints. We proposed an online algorithm, the sLLF algorithm, that decides on the current charging rates based on only the information up to the current time. We characterized and analyzed the performance of the sLLF algorithm analytically and numerically utilizing the resource augmentation framework, where we demonstrated the first 
application of the framework for evaluating EV charging algorithms. Here, we also extend the resource augmentation framework for heterogeneous processors with a time-varying number application.

Our numerical experiments with real-world datasets showed that our algorithm has a significantly higher rate of generating feasible EV charging than several other common EV charging algorithms. We showed that our sLLF algorithm is able to maintain a high success rate. The algorithm also shows its potential benefits against a large variety of EVs' sojourn time that common in a real-world application and against a high urgency scheduling environment such as in some public charging stations; more study is needed. By finding feasible EV charging schedules using only a small augmentation that is also significantly less than the theoretical upper bound, our proposed algorithm (sLLF) can significantly reduce the infrastructural cost for $\mathrm{EV}$ charging facilities. Among the algorithms that achieve the highest success rate (i.e., the sLLF, LLF, and OLP algorithms), our sLLF algorithm does not exhibit undesirable oscillations such found in the LLF algorithm and computationally cheaper than the OLP algorithm.

\section{REFERENCES}

[1] O. Sundstrm and C. Binding, "Planning electric-drive vehicle charging under constrained grid conditions," in 2010 Int. Conf. on Power System Technology, 2010, pp. 1-6. doi: 10.1109/POWERCON.2010.5666620

[2] P. Richardson, D. Flynn, and A. Keane, "Local versus centralized charging strategies for electric vehicles in low voltage distribution systems," IEEE Trans. on Smart Grid, vol. 3, no. 2, pp. 1020-1028, 2012. doi: 10.1109/TSG.2012.2185523

[3] L. Gan, U. Topcu, and S. H. Low, "Optimal decentralized protocol for electric vehicle charging," IEEE Trans. on Power Systems, vol. 28, no. 2, pp. 940-951, 2013. doi: 10.1109/TPWRS.2012.2210288

[4] Z. Ma, D. S. Callaway, and I. A. Hiskens, "Decentralized charging control of large populations of plug-in electric vehicles," IEEE Trans. on Control Systems Technology, vol. 21, no. 1, pp. 67-78, 2013. doi: 10.1109/TCST.2011.2174059

[5] N. Chen, C. W. Tan, and T. Q. S. Quek, "Electric vehicle charging in smart grid: Optimality and valley-filling algorithms," IEEE J. of Selected Topics in Signal Processing, vol. 8, no. 6, pp. 1073-1083, 2014. doi: 10.1109/JSTSP.2014.2334275

[6] L. Gan, A. Wierman, U. Topcu, N. Chen, and S. H. Low, "Realtime deferrable load control: Handling the uncertainties of renewable generation," in Proc. of the 4th Int. Conf. on Future Energy Systems, ser. e-Energy '13. New York, NY, USA: ACM, 2013, p. 113124. doi: 10.1145/2487166.2487179. ISBN 9781450320528

[7] S. Chen, Y. Ji, and L. Tong, "Large scale charging of electric vehicles," in 2012 IEEE Power and Energy Soc. General Meeting, 2012, pp. 1-9. doi: 10.1109/PESGM.2012.6345497

[8] Z. Yu, Y. Xu, and L. Tong, "Deadline scheduling as restless bandits," in 54th Annu. Allerton Conf. on Communication, Control, and Computing, 2016, pp. 733-737. doi: 10.1109/ALLERTON.2016.7852304

[9] S. Stein, E. Gerding, V. Robu, and N. R. Jennings, "A modelbased online mechanism with pre-commitment and its application to electric vehicle charging," in Proc. of the 11th Int. Conf. on Autonomous Agents and Multiagent Systems, ser. AAMAS '12, Richland, SC, 2012, p. 669676. ISBN 0981738125. [Online]. Available: http://dl.acm.org/citation.cfm?id=2343776.2343792

[10] F. Kong, Q. Xiang, L. Kong, and X. Liu, "On-line eventdriven scheduling for electric vehicle charging via park-and-charge," in 2016 IEEE Real-Time Systems Symp., 2016, pp. 69-78. doi: 10.1109/RTSS.2016.016

[11] L. Guo, K. F. Erliksson, and S. H. Low, "Optimal online adaptive electric vehicle charging," in 2017 IEEE Power Energy Society General Meeting, 2017, pp. 1-5. doi: 10.1109/PESGM.2017.8274166

[12] M. Alonso, H. Amaris, J. G. Germain, and J. M. Galan, "Optimal charging scheduling of electric vehicles in smart grids by heuristic algorithms," Energies, vol. 7, no. 4, pp. 2449-2475, 2014. doi: $10.3390 /$ en7042449
[13] M. Amjad, A. Ahmad, M. H. Rehmani, and T. Umer, "A review of evs charging: From the perspective of energy optimization, optimization approaches, and charging techniques," Transp. Res. Part D: Transport and Environment, vol. 62, pp. 386-417, 2018. doi: 10.1016/j.trd.2018.03.006

[14] T. Mao, X. Zhang, and B. Zhou, "Intelligent energy management algorithms for ev-charging scheduling with consideration of multiple ev charging modes," Energies, vol. 12, no. 2, 2019. doi: $10.3390 /$ en 12020265

[15] M. Elmehdi and M. Abdelilah, "Genetic algorithm for optimal charge scheduling of electric vehicle fleet," in Proc. of the 2nd Int. Conf. on Networking, Information Systems \& Security, ser. NISS19. New York, NY, USA: ACM, 2019, pp. 1-7. doi: 10.1145/3320326.3320329. ISBN 9781450366458

[16] M. E. Kabir, C. Assi, M. H. K. Tushar, and J. Yan, "Optimal scheduling of ev charging at a solar power-based charging station," IEEE Syst. J., vol. 14, no. 3, pp. 4221-4231, 2020. doi: 10.1109/JSYST.2020.2968270

[17] A. Subramanian, M. J. Garcia, D. S. Callaway, K. Poolla, and P. Varaiya, "Real-time scheduling of distributed resources," IEEE Trans. on Smart Grid, vol. 4, no. 4, pp. 2122-2130, 2013. doi: 10.1109/TSG.2013.2262508

[18] J. A. Stankovic, M. Spuri, K. Ramamritham, and G. C. Buttazzo, Deadline Scheduling for Real-Time Systems. Boston, MA: Springer US, 1998. ISBN 978-1-4613-7530-2

[19] P. Whittle, "Restless bandits: Activity allocation in a changing world," J. of Applied Probability, vol. 25, pp. 287-298, 1988. [Online]. Available: http://www.jstor.org/stable/3214163

[20] M. Brenna, F. Foiadelli, C. Leone, and M. Longo, "Electric vehicles charging technology review and optimal size estimation," J. of Electrical Engineering \& Technology, vol. 15, no. 6, pp. 2539-2552, Nov 2020. doi: $10.1007 / \mathrm{s} 42835-020-00547-\mathrm{x}$

[21] B. Kalyanasundaram and K. Pruhs, "Speed is as powerful as clairvoyance," J. of the ACM, vol. 47, no. 4, p. 617643, Jul. 2000. doi: $10.1145 / 347476.347479$

[22] Phillips, Stein, Torng, and Wein, "Optimal time-critical scheduling via resource augmentation," Algorithmica, vol. 32, no. 2, pp. 163-200, Feb 2002. doi: 10.1007/s00453-001-0068-9

[23] S. Im, J. Kulkarni, and K. Munagala, "Competitive algorithms from competitive equilibria: Non-clairvoyant scheduling under polyhedral constraints," J. of the ACM, vol. 65, no. 1, Dec. 2017. doi: 10.1145/3136754

[24] S. Im, J. Kulkarni, K. Munagala, and K. Pruhs, "Selfishmigrate: A scalable algorithm for non-clairvoyantly scheduling heterogeneous processors," in 2014 IEEE 55th Annu. Symp. on Foundations of Computer Science, 2014, pp. 531-540. doi: 10.1109/FOCS.2014.63

[25] C. L. Liu and J. W. Layland, "Scheduling algorithms for multiprogramming in a hard-real-time environment," J. of the ACM, vol. 20, no. 1, p. 4661, Jan. 1973. doi: 10.1145/321738.321743

[26] M. Dertouzos and A. Mok, "Multiprocessor online scheduling of hardreal-time tasks," IEEE Trans. on Software Engineering, vol. 15, no. 12, pp. 1497-1506, 1989. doi: 10.1109/32.58762

[27] R. I. Davis and A. Burns, "A survey of hard real-time scheduling for multiprocessor systems," ACM Computing Survey, vol. 43, no. 4, Oct. 2011. doi: $10.1145 / 1978802.1978814$

[28] G. Lee, T. Lee, Z. Low, S. H. Low, and C. Ortega, "Adaptive charging network for electric vehicles," in 2016 IEEE Global Conf. on Signal and Information Processing, 2016, pp. 891-895. doi: 10.1109/GlobalSIP.2016.7905971

[29] Z. J. Lee, D. Chang, C. Jin, G. S. Lee, R. Lee, T. Lee, and S. H. Low, "Large-scale adaptive electric vehicle charging," in 2018 IEEE Global Conference on Signal and Information Processing, 2018, pp. 863-864. doi: 10.1109/GlobalSIP.2018.8646472

[30] Z. J. Lee, T. Li, and S. H. Low, "Acn-data: Analysis and applications of an open ev charging dataset," in Proc.s of the 10th ACM Int. Conf. on Future Energy Systems, ser. e-Energy '19. New York, NY, USA: ACM, 2019, p. 139149. doi: 10.1145/3307772.3328313. ISBN 9781450366717

[31] A. K.-L. Mok, "Fundamental Design Problems of Distributed Systems for The Hard-Real-Time Environment," Ph.D. dissertation, Massachusetts Institute of Technology, 1983. [Online]. Available: http://hdl.handle.net/1721.1/15670

[32] N. Chen, C. Kurniawan, Y. Nakahira, L. Chen, and S. H. Low, "Smoothed least-laxity-first algorithm for ev charging," 2021. [Online]. Available: https://arxiv.org/abs/2102.08610

[33] J. Kay and P. Lauder, "A fair share scheduler," Commun. of ACM, vol. 31, no. 1 , p. 4455 , Jan. 1988. doi: 10.1145/35043.35047

[34] S. Jiang, Z. Song, O. Weinstein, and H. Zhang, "Faster dynamic matrix inverse for faster lps," 2020. [Online]. Available: https: //arxiv.org/abs/2004.07470 


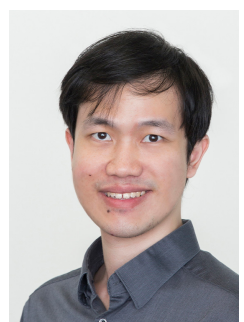

Niangjun Chen received his B.S. in Computer Science at the University of Cambridge, and his M.S and Ph.D. in Computer Science at the California Institute of Technology. His research interests include optimization, machine learning, game theory, and their applications to complex systems such as smart grids, data centers, and transport. After graduation in 2017, he joined the Institute of High Performance Computing as a research scientist working on the optimization of logistics and the modeling and simulation of the transport systems. Since September 2020, he is an Assistant Professor at the Singapore University of Technology and Design. He has a joint appointment at the Institute for High Performance Computing at the Agency for Science, Technology, and Research (A*STAR).

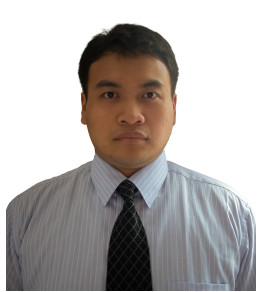

Christian Kurniawan received a B.Sc. degree in Mathematics from Brigham Young University, Hawaii campus in 2014 and an M.S. degree in Mechanical Engineering with an emphasis in computational grain boundary from Brigham Young University main campus in 2018 . His current research interest includes applications of optimization techniques and inverse problem theory in various computational engineering problems and autonomous systems.

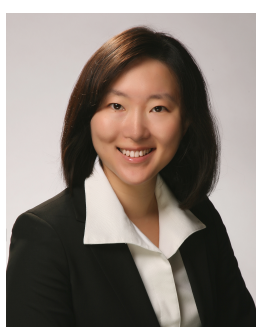

Yorie Nakahira is an Assistant Professor of Electrical and Computer Engineering at Carnegie Mellon University. She received a Ph.D. in Control and Dynamical Systems from California Institute of Technology, and a Bachelors degree from Tokyo Institute of Technology. Her research interests include optimization, control, and information theory with applications in neuroscience and biology.

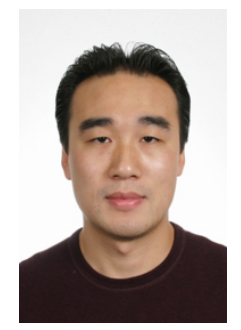

Lijun Chen (M'05) is an Assistant Professor of Computer Science at University of Colorado at Boulder. He received a Ph.D. in Control and Dynamical Systems from California Institute of Technology in 2007. He was a co-recipient of the Best Paper Award at the IEEE International Conference on Mobile Ad-hoc and Sensor Systems (MASS) in 2007. His current research interests include optimization and control of networked systems and autonomous systems, distributed optimization and control, machine learning and its integration with control, and game theory and its engineering application. He was an editor for the IEEE Transactions on Communications 2015-2021, and is editor for the IEEE Control Systems Society Conference Editorial Board. He served as the chair of the Special Interest Group in ICT-Enabled Transactive Energy and Grid Economics of IEEE ComSoc Smart Grid Communications Emerging Technical Subcommittee 2017-2019.

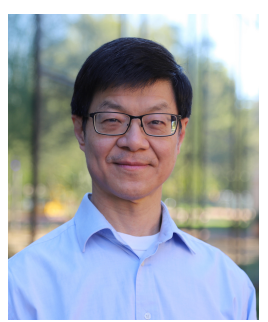

Steven H. Low (F2008) is the F. J. Gilloon Professor of the Department of Computing \& Mathematical Sciences and the Department of Electrical Engineering at Caltech. Before that, he was with AT\&T Bell Laboratories, Murray Hill, NJ, and the University of Melbourne, Australia. He has held honorary/chaired professorship in Australia, China and Taiwan. He was a co-recipient of IEEE best paper awards, an awardee of the IEEE INFOCOM Achievement Award and the ACM SIGMETRICS Test of Time Award, and is a Fellow of IEEE, ACM, and CSEE. He was well-known for work on Internet congestion control and semidefinite relaxation of optimal power flow problems in smart grid. His research on networks has been accelerating more than 1TB of Internet traffic every second since 2014. His research on smart grid is providing large-scale cost effective electric vehicle charging to workplaces. He received his B.S. from Cornell and $\mathrm{PhD}$ from Berkeley, both in EE. 